\title{
Mutations in CHMP4C cause dilated cardiomyopathy via dysregulation of autophagy
}

wei Zhou ${ }^{1}$, Lu Tang ${ }^{2}$, Yingying Jiang ${ }^{2}$, xuejie li ${ }^{1}$, minmin $\operatorname{sun}^{1}$, haiyan chen ${ }^{1}$, jie cui $^{2}$, shifang $\operatorname{shan}^{3}$, Bo Yuan ${ }^{3}$, shengmei qin ${ }^{2}$, wenqing zhu ${ }^{1}$, weipeng zhao ${ }^{2}$, Cuizhen pan ${ }^{2}$, Xianghong $\mathrm{Shu}^{2}$, xiaolin wang ${ }^{1}$, Zilong $\mathrm{Qiu}^{3}$, and Junbo $\mathrm{Ge}^{1}$

${ }^{1}$ Fudan University

${ }^{2}$ Zhongshan Hospital Fudan University

${ }^{3}$ Chinese Academy of Sciences Shanghai Branch

May 5, 2020

\begin{abstract}
Gene mutations have been implicated in DCM. However, due to the difficulty of clinical genetic diagnosis, additional causal genes potentially related to DCM remain to be discovered. We screened for gene mutations in more than 400 cases from families with hereditary cardiovascular disease using whole-exome sequencing and then validated the biological functions of CHMP4C mutations in zebrafish models. To further assess the mechanism of CHMP4C mutations, we determined the potential signaling pathway in a cell line.We identified via whole-exome sequencing CHMP4C variants that segregated with DCM in four families among a total of 411 families. We further validated the function of CHMP4C in heart function in zebrafish models and found that overexpression of CHMP4C variants resulted in cardiac malformation, pericardial edema and an increased heart rate, consistent with CHMP4C mutation-associated findings in DCM patients. Furthermore, mutations in CHMP4C impaired autophagy and activated apoptosis in HEK293T cells, suggesting that the molecular mechanism of CHMP4C is involved in heart development.CHMP4C is a novel candidate gene causing DCM and may play a critical role in cardiac development by regulating autophagy.
\end{abstract}

\section{Introduction}

Dilated cardiomyopathy (DCM) is a primary myocardial disease of unknown cause (Reichart, et al., 2019)that is characterized by ventricular enlargement and ventricular systolic dysfunction(Pasotti, et al., 2008). DCM exhibits obvious heterogeneity, from a lack of symptoms to arrhythmia and heart failure. Early arrhythmia is a common condition(Watkins, et al., 2011) that may progressively become aggravated, and death can occur at any stage of the disease. The most serious complications are heart failure and sudden death(Hershberger and Siegfried, 2011). Based on clinical studies, the incidence of DCM in the population is approximately $1 / 2500$ (McNally, et al., 2013). Moreover, DCM and other cardiomyopathies, such as hypertrophic cardiomyopathy, right ventricular cardiomyopathy, and ion channel disease, intersect with regard to clinical manifestations and gene mutations(Dellefave and McNally, 2010; Gentile, et al., 2019; Kimura, 2016).

Gene mutations have been implicated in DCM. Indeed, genetic screening indicates that approximately $35 \%$ of cases are primarily caused by mutations, including those in genes related to the cardiomyocyte cytoskeleton, nuclear membrane, and ion channels(Bollen, et al., 2017; Costa, 2019). However, due to the difficulty of clinical genetic diagnosis, other causal genes potentially related to DCM remain to be discovered(Bagnall, et al., 2011; Lakdawala, et al., 2013). Therefore, we screened for pathogenic genes in patients with clinically diagnosed cardiomyopathies using whole-exome sequencing. 
Here, we report the identification of $\mathrm{CHMP}_{4} \mathrm{C}$ (which encodes a protein of the endosomal sorting complex required for transport) (Pike, et al., 2016) variants segregating in four DCM families from among a total of 411 families by whole-exome sequencing. We further validated the function of CHMP4C in heart function in zebrafish models and found that overexpression of $\mathrm{CHMP}_{4} \mathrm{C}$ variants resulted in cardiac malformation, pericardial edema and an increased heart rate, consistent with CHMP4C mutation-associated findings in DCM patients. Furthermore, we found that mutations in $C H M P 4 C$ impaired autophagy and activated apoptosis in HEK293T cells, suggesting that the molecular mechanism of CHMP4C is involved in heart development. Together, these findings suggest that CHMP4C is a novel candidate gene causing DCM and may play a critical role in cardiac development by regulating autophagy.

\section{Methods}

\section{Subjects}

A total of 411 families were enrolled, and each family included patients and their relatives. We performed whole-exome sequencing on cardiomyopathy probands; if potential candidate genes were identified, wholeexome sequencing was performed for their relatives. The entire family was then examined for cosegregation and possible pathogenic genes. Sanger sequencing verification was performed for the families of probands carrying mutations in causative genes. The study was approved by the Ethics Committee of Zhongshan Hospital, Fudan University (No. B2016-016(2)R).

Sequencing data analysis

Alignment, variant calling, and quality control

We sequenced the whole exome of cardiomyopathy samples. Genomic DNA was used to produce each exomecaptured library with Agilent SureSelect V5, MGI V4 and IDT .xGen Exome Research Panel. Subsequently, each captured library was independently sequenced using the BGISEQ-500 or Illumina HiSeq 2000 platform. Each sample was designed to obtain high-quality bases with an average coverage of $>100 \times$. Raw sequence reads were mapped to Human Genome Assembly GRCh37. GATK best practices for joint analysis for the multisample approach were adopted in our study. First, we generated gVCF files for each sample using HaplotypeCaller and combined all gVCF files using GenotypeGVCFs to produce a combined variant VCF file. Some basic filtering parameters from the GATK best practices workflow were applied to filter the variants. Second, individual genotypes were input from the sequencing data using BEAGLE with default parameters for primary SNP calls. Such inputted genotypes are more accurate than are genotypes called directly from sequencing data, especially for SNPs with low sequencing depth, as the linkage disequilibrium information of neighboring variants is incorporated into the imputation model. Finally, we employed a series of variant filters to remove false or low-quality variants, including parameters of sequencing depth, mappability score, strand bias, allelic balance, length homopolymer run, base quality and Hardy-Weinberg equilibrium.

\section{Gene-based analysis}

The results were filtered against in-house and public databases, as well as appropriate genetic models (those with autosomal dominant mutations). Additional automatic filtering was applied to remove sequencing artifacts. We adopted a simple collapse method by counting the individuals who carried rare variants (minimum allele frequency, MAF [?]5\%) at the gene level. To best identify putatively damaging single-nucleotide variants (SNVs), five protein prediction algorithms (LRT score (ref), MutationTaster (ref), PolyPhen-2 HumDiv (ref), PolyPhen-2 HumVar (ref) and SIFT (ref)) were used to examine nonsynonymous SNVs. These variant groups are as follows: (1) nonsynonymous (missense, splicing, nonsense and indel frameshift variants); (2) a 'deleterious (PolyPhen)' set consisting of missense variants annotated as 'possibly damaging' or 'probably damaging' by PolyPhen-2 HumDiv software; (3) a 'deleterious (broad)' set consisting of missense variants annotated as 'deleterious' by at least one of the above five protein prediction algorithms; (4) a 'deleterious (strict)' set consisting of missense variants annotated as 'deleterious' by all of the above five protein prediction algorithms; and (5) 'disruptive' mutations only (nonsense, splice site or indel frameshift). We extracted SNVs with an MAF $<5 \%$ for relevant gene-based analysis after collapsing all 
nonsynonymous mutations. Evolutionary conservation analysis was performed with various species using Clustal W (http://www.genome.jp/tools/clustalw/) and Weblogo (http://weblogo.berkeley.edu/logo.cgi). Protein structure of the mutated proteins identified in this study was predicted using the SWISS-MODEL (https://swissmodel.expasy.org/) online program.

Sanger sequencing and analyses

Sanger sequencing was performed on DNA samples from each family's proband for genotype confirmation and on samples from the remaining family members for familial cosegregation analyses.

Construction of the zebrafish mutant model

The expression plasmid pXT7-CHMP4C (wt/mut) was constructed, and then the CHMP4C in vitro transcription template was prepared. The plasmids were linearized using a single enzyme (XbaI) in a reaction containing FastDigest Green Buffer $(5.0 \mu \mathrm{l})+\mathrm{XbaI}(2.5 \mu \mathrm{l})+$ pXT7-CHMP4C (WT/MUT) $(2.0 \mu \mathrm{g})$ and Milli-Q $\mathrm{H}_{2} \mathrm{O}$ to $50 \mu \mathrm{l}$ in a $37^{\circ} \mathrm{C}$ water bath for 2 hours. The in vitro transcription templates were purified, and mMESSAGE mMACHINE T7 Ultra Transcription Kit (Ambion) was used as follows: $2 \times$ NTP/ARCA $(10.0 \mu \mathrm{l})+10 \times \mathrm{T} 7$ Reaction Buffer $(2.0 \mu \mathrm{l})+C H M P 4 C$ (WT/MUT) $(1.0 \mu \mathrm{g})+\mathrm{T} 7$ Enzyme Mix $(2.0 \mu \mathrm{l})$ and nuclease-free water to $20.0 \mu \mathrm{l}$ was incubated in a $37^{\circ} \mathrm{C}$ water bath for 2 hours. Next, $1 \mu \mathrm{l}$ of TURBO DNase I was added, and the sample was incubated at $37^{\circ} \mathrm{C}$ for $15 \mathrm{~min}$. The DNA template was removed, and capped $C H M P 4 C$ (WT/MUT) mRNA was obtained, followed by a reaction containing T7 Ultra Reaction $(18.0 \mu \mathrm{l})+5 \times$ E-PAP Buffer $(20.0 \mu \mathrm{l})+25 \mathrm{mM} \mathrm{MnCl2}(10.0 \mu \mathrm{l})+$ ATP Solution $(10.0 \mu \mathrm{l})+\mathrm{E}$-PAP $(4.0 \mu \mathrm{l})$ + nuclease-free water $(38.0 \mu \mathrm{l})$ in a $37^{\circ} \mathrm{C}$ water bath for $30-45 \mathrm{~min}$. The quality, purity, and concentration of the in vitro transcription mRNA products were assessed by electrophoresis. Fertilized eggs of the zebrafish $\mathrm{AB}$ strain were collected. CHMP4C mRNA (WT and E163G) at final concentrations of 50, 100 and 200 $\mathrm{ng} / \mu \mathrm{l}$ or CHMP4C mRNA (WT and S204L) at final concentrations of 25, 50 and $100 \mathrm{ng} / \mu \mathrm{l}$ was injected microscopically into the fertilized eggs (at least 200 injections per group) using an injection volume of $1 \mathrm{nl}$ per embryo; the control groups were not injected. The embryos were observed under a stereomicroscope at $50 \mathrm{hpf}$ and $120 \mathrm{hpf}$, and the heart rate, cardiac malformation and pericardial edema were observed and recorded.

Culture and transfection of HEK293T cells

A sample of $5 \times 10^{5}$ HEK293T cells was inoculated into a 12-well plate and grown to approximately $60 \%-70 \%$ confluence in DMEM with $10 \%(\mathrm{v} / \mathrm{v}) \mathrm{FBS}$ in a $\mathrm{CO}_{2}$ incubator with $5 \%$. Two micrograms of the FUGWCHMP $4 \mathrm{C}$ wildtype, mutant and control plasmids were diluted with $50 \mu \mathrm{l}$ Opti-MEM and mixed gently, and $1 \mu \mathrm{l}$ Lipofectamine 2000 was diluted with $50 \mu \mathrm{l}$ Opti-MEM and incubated for $5 \mathrm{~min}$ at room temperature; these two mixtures were gently mixed and kept at room temperature for $20 \mathrm{~min}$, after which $100 \mu \mathrm{l}$ was added to each well. Fresh medium was added after 4 hours. After 48 hours, one group was treated with medium lacking serum; the control cells were grown with serum. Each group of cells was collected for further analyses.

Library preparation and data analyses for zebrafish RNA-seq

Fertilized zebrafish eggs were microinjected with $50 \mathrm{pg} / \mathrm{nl}$ (in a total volume of $1 \mathrm{nl}$ ) CHMP4C wildtype mRNA and mutant mRNA. A blank control group was set up; the number of embryos injected per group was approximately 200. The injected and control embryos were cultured as usual. After embryo development to $50 \mathrm{hpf}, 30$ embryos with relatively normal development and control embryos were selected. After the addition of TRIzol, the embryos were immediately frozen at $-80^{\circ} \mathrm{C}$ and sent for transcriptome sequencing. Total RNA was extracted from the embryo, and quality control was performed before sequencing.

Bioinformatics analysis of transcriptome data was performed as follows: library preparation for RNA-seq was performed using NEBNext Ultra Directional RNA Library Prep Kit for Illumina (E7420S, NEB) according to the manufacturer's instructions and further subjected to Illumina HiSeq 2000 for paired-end sequencing. The raw sequencing reads were first processed to remove bases of low quality, and only the first $35 \mathrm{bp}$ from two ends of each read was used for downstream analysis. 
Isolation and culture of primary cardiomyocytes

The thoracic cavities of 15 newborn rats (1-3 days old) were disinfected with $75 \%$ alcohol and cut open, and the ventricular tissue was removed; the tissue was washed with PBS, and excess tissue around the heart and blood clots were removed. The tissue was washed again with PBS, and the supernatant was aspirated with a pipette. Then, the heart tissue was cut to a size of $1 \mathrm{~mm}^{3}$, placed in a $50 \mathrm{ml}$ beaker with a magnetic stirrer, and trypsin was added to a total volume of approximately $15 \mathrm{ml}$. The beaker was covered with foil and placed into a $37^{\circ} \mathrm{C}$ water bath with a magnetic stirrer at $60 \mathrm{r} / \mathrm{min}$ for $10 \mathrm{~min}$. Fifteen milliliters of trypsin was added again, and the samples were digested for $10 \mathrm{~min}$. After centrifugation, the supernatant liquid was carefully removed, and the bottom cell pellet was resuspended in 3-4 $\mathrm{ml}$ of cell culture medium. The cell pellet was then broken up as much as possible with a sterile pipette and was then pipetted into another centrifuge tube. The above steps were repeated 6-7 times, and the digested cardiomyocytes were collected and centrifuged at $1000 \mathrm{r} / \mathrm{min}$ for $5 \mathrm{~min}$. The resuspended cells were transferred to a $100 \mathrm{~mm}$ tissue culture dish and placed in a $37^{\circ} \mathrm{C}$ cell incubator for 1.5-2 hours. Subsequently, the cell density was adjusted to $1 \mathrm{x}$ $10^{6}$ cells $/ \mathrm{ml}$ with primary cardiomyocyte culture medium and added to a sterile 6 -well culture plate, and 2 $\mathrm{ml}$ of medium was added to each well. After 24 hours, the cultured primary cardiomyocytes were washed once with prewarmed medium so that unattached cells could be washed away. The fresh cell culture medium was replaced, and further treatments were applied after 48 hours or 72 hours of further cultivation according to the requirements of the experiment.

Western blot analysis

Cell protein sample extraction was performed as follows. The culture medium was removed, and 1X SDS sample buffer was added. The cells were scraped from the plates and incubated for $15 \mathrm{~min}$ at $100^{\circ} \mathrm{C}$. Equal amounts of protein were separated by SDS-PAGE, along with a molecular weight marker; $90 \mathrm{~V}$ was applied for the stacking gel and $120 \mathrm{~V}$ for the resolving gel. A PVDF membrane was activated with methanol for $1 \mathrm{~min}$ and rinsed with transfer buffer. The proteins were transferred from the gel to the membrane for 90 min at $200 \mathrm{~mA}$, followed by blocking of the membrane for 2 hours at room temperature. The membrane was incubated overnight with appropriate dilutions of primary antibodies in blocking buffer at $4^{\circ} \mathrm{C}$ and washed three times with TBST for $15 \mathrm{~min}$ each. The membrane was incubated with the recommended dilution of conjugated secondary antibody in blocking buffer at room temperature for 2 hours and washed three times with TBST for 15 min each. The chemiluminescent substrate was applied to the blot according to the SuperSignal West Pico instructions (34080, Thermo Fisher Scientific), and the chemiluminescent signals were captured using film.

Antibodies (name, no., brand)

Antibodies against the following were used in this study: m-TOR (2983, Cell Signaling Technology); Sqstm1/p62 (ab56416, Abcam); Atg5-atg12 (2011, Cell Signaling Technology); LC3 (M186-3, MBL); AKT (4691, Cell Signaling Technology); p-AKT (4060, Cell Signaling Technology); CHMP4C (ab155668, Abcam); Bcl-2 (15071, Cell Signaling Technology); Bax (5023, Cell Signaling Technology); Caspase-3 (9662, Cell Signaling Technology); Caspase-9 (9508, Cell Signaling Technology); and $\beta$-Actin (P30002, Abmart).

Immunofluorescence

After 48 hours of transfection, cells were fixed with $4 \%$ polyformaldehyde for 2 hours. Residual polyformaldehyde was removed with PBS, and the cell membrane was permeabilized with $0.1 \%$ Triton X-100 at room temperature for 15 min. Blocking buffer (3\% BSA and 0.1\% Triton X-100 soluble in PBS) was added, and the samples were incubated for 1 hour at room temperature. Mouse anti-LC3 (1:500) was diluted in blocking buffer and incubated at room temperature for 2 hours, and the cells were washed 3 times with PBS for 10 min. Donkey anti-mouse IgG (1:1000) was then added and incubated at room temperature for 1 hour. The cells were washed three times with PBS for 5 min. DAPI was added and incubated in the dark at room temperature for $10 \mathrm{~min}$. Fluorescent images were captured under a confocal microscope.

Transmission electron microscopy sample preparation and photography 
In total, HEK293T cells $\left(1 \times 10^{6}\right)$ were collected, fixed with $2.5 \%$ dialdehyde for 2 hours and rinsed with 0.1 $\mathrm{M}$ phosphoric acid (15 min, 3 times). The liquid was fixed with $1 \%$ picric acid for 2 hours and then rinsed with $0.1 \mathrm{M}$ phosphoric acid (15 min, 3 times). The samples were dehydrated with $50 \%$ ethanol for $15-20 \mathrm{~min}$, $70 \%$ ethanol for 15-20 min, 90\% ethanol for 15-20 min, 90\% ethanol and 90\% acetone (1:1) for 15-20 min, and $90 \%$ acetone for 15-20 min. The above procedure was carried out at $4^{\circ} \mathrm{C}$, followed by $100 \%$ acetone at room temperature for 15-20 min. Pure acetone and encapsulation (2:1) were performed at room temperature for 3-4 hours, at (1:2) room temperature overnight, and at $37^{\circ} \mathrm{C}$ for $2-3$ hours. Curing was performed in a $37^{\circ} \mathrm{C}$ oven overnight, in a $45^{\circ} \mathrm{C}$ oven for 12 hours, and in a $60^{\circ} \mathrm{C}$ oven for 48 hours. A microtome was used to slice sections at a thickness of $70 \mathrm{~nm}$, which were dyed with $3 \%$ uranium acetate-lead with fenthion acid. Photographs were obtained using a Philips CM 120 microscope.

Statistics and reproducibility

For experiments including multiple comparisons, $\mathrm{P}$ values refer to the results of one-way analysis of variance (ANOVA) followed by Tukey's post hoc test. $\mathrm{P}$ values of $<0.05$ were considered significant. $\mathrm{P}$ values are provided as exact figures where possible or are otherwise reported as a range in a single figure or panel when a large number of comparisons were performed. Data are shown as the mean + - sem or sd.

\section{Results}

\section{Identification of CHMP4C mutations in DCM trios}

We identified that CHMP4C mutations cosegregated with DCM patients from four families. We identified that $C H M P 4 C$ mutations cosegregated with DCM patients from four families. The proband of Family 1 is a Han female, 58 years old (III4, Fig. 1a), who visited our hospital with repeated recurrent syncopy and fatigue. Electrocardiogram (ECG) showed atrial fibrillation and left bundle-branch block. Echocardiography and MRI suggested enlargement of the entire heart. The left ventricular systolic function was globally reduced, and the left ventricular ejection fraction (LVEF) was 32\% (Supplemental Figure 1a). Serum creatine kinase and troponin $\mathrm{T}$ were significantly higher than normal. The patient had no previous history of hypertension, coronary heart disease or diabetes (III4). She (III4) was diagnosed with DCM, followed by prescribed medication and implantation of a pacemaker. The family history of the proband revealed more family members with abnormal cardiac function and malignant cardiovascular events. Some of her family members also had cardiac dysfunction by clinical examination. Whole-exome sequencing was performed for both affected family members (III4, III7, III10, Fig. 1a) and unaffected family members (III1, III9, III13, Fig. 1a), followed by bioinformatics analysis.

We found that a heterozygous mutation in the fourth exon of the CHMP4C gene, c.488 A>G, p.Glu163Gly, cosegregated with the affected members, suggesting that this CHMP4C mutation is associated with DCM in this family. This mutation site is rare (gnomAD 34/282350, 0.0001204), and SIFT and Polyphen2 software predict damage to the CHMP4C protein. Furthermore, this mutation site is in a conserved region across multiple specifies (Fig. 1e). Based on Sanger sequencing of the genomes of the other family members, the mutation is consistent with Mendelian genetic characteristics (Supplementary Fig. 1a).

After analyzing this $C H M P 4 C$ mutation (c.488 A>G, p.Glu163Gly) in Family 1, we hypothesized that $C H M P 4 C$ may be a new cardiomyopathy causative gene. Therefore, we screened for $C H M P 4 C$ gene mutations in more than 400 cases from families with hereditary cardiovascular disease using whole-exome sequencing (214 cases of hypertrophic cardiomyopathy, 110 cases of DCM, 12 cases of right ventricular cardiomyopathy, 75 cases of other cardiovascular diseases), especially in families that did not have a mutation in a known disease-causing gene. Two new mutation sites were found in three families: Family 2 (DCM, Figure 1b) and Family 3 (DCM, Figure 1c), both of which carried a CHMP 4 C missense mutation, c.C611T, p.S204L (gnomAD, 21/282456, 0.00007435); Family 4 (DCM, Figure 1d) carried a frameshift mutation, c.73_104delGCCCTGGTCCGACTTCGGGAGACTGAG GAGAT, p.L26Gfs*26 (gnomAD, 1/213196, 0.000004691) (Table 1).

The proband of Family 2 (II-2) was a 49-year-old Han woman with occasional chest tightness. ECG showed 
frequent ventricular premature beats, and echocardiography revealed a slight decrease in LVEF (47\%). There was no abnormality on coronary angiography, no history of hypertension or diabetes mellitus, and no significant improvement after routine medical treatment (Supplemental Figure 1b). Sanger validation for the family demonstrated that the patient's mother (I-2), brother (II-3) and younger brother's child (III-2) all carried the pathogenic mutation. The father (I-1), 74 years old, had normal cardiac function; the mother (I2 ), also 74 years old, had a history of cardiac dysfunction. The younger brother, 46 years old, had premature beats and ST changes on ECG, though no heart abnormalities were found in his child, who was 22 years old.

In Family 3, we first found a mutant in $L M N A$ (c.961 C > T, p.Arg321Ter) in affected family members during initial gene screening in 2016 (Figure 1c). A 44-year-old patient (III-11) exhibited mild left ventricular enlargement with paroxysmal tachycardia during the follow-up visit in 2019. In 2016, the patient's cardiac function was in a critical state (occasional premature ventricular contraction and ST changes, normal cardiac systolic function, LVEF was $56 \%$ (Supplemental Figure 1c)). However, he did not carry the LMNA mutation. After reanalysis, we found that III-7, III-11, and III-17 also carried the CHMP 4 C p.S204L mutation. The clinical manifestations of III-7 and III-17 occurred earlier than did those of III-11, and the degree of heart failure was more serious (both patients were younger than 40 years old when pacemakers were implanted). This clinical evidence strongly suggests that the CHMP 4 C p.S204L mutation contributes to DCM.

Echocardiography examination of the proband of Family 4 (II-5) showed that the heart was globally enlarged; the left and right ventricular contractile function was also weakened $(\mathrm{LVEF}=24 \%)$ (Figure 1d). We found a potential pathogenic mutation in $T T N($ c. $9220 \mathrm{C}>\mathrm{T}$, p.Arg3074*) in ensuing screening. However, only the patient and her daughter carried the pathogenic mutation, whereas the proband's sister did not. We speculated that other pathogenic mutations may exist, and after refiltration, we found that all three patients carried a CHMP 4 C mutation (c.73_104del, p.L26Gfs*26). Moreover, those patients who also carried the TTN mutation had a more severe clinical manifestation and an earlier onset (Supplemental Figure 1d and Table 1).

\section{Validation of the biological functions of CHMP4C mutations in zebrafish models}

Considering that these recurrent $C H M P 4 C$ mutations are associated with DCM, the truncated mutant proteins are likely to have damaging effects; however, it is unclear whether normal protein function is affected by CHMP4C-E163G and CHMP4C-S204L. To verify whether these two missense mutants affect protein functions, we performed in vivoand in vitro studies.

To investigate whether CHMP4C mutations may affect heart developmentin vivo, we used a zebrafish model to examine the effect of CHMP4C-E163G and CHMP4C-S204L mutant proteins on cardiac function. Based on the zebrafish phenotype as a function of the concentration injected, we selected $50 \mathrm{ng} / \mu \mathrm{l}$ and $120 \mathrm{hpf}$ for further analysis. In zebrafish injected with CHMP4C-E163G and CHMP4C-S204L, we observed myocardial tissue morphology by section staining and the myocardial tissue microstructure by electron microscopy (Figure 2a,b). The proportion of cardiac malformation and pericardial edema in zebrafish injected with CHMP4C-E163G and CHMP4C-S204L mutations were significantly increased compared with those in the wildtype and control groups, as was the heart rate (Figure 2c-h). There was no significant difference between the wildtype and control groups (Figure 2c-h). Similar changes were found by myocardial tissue staining.

Furthermore, we observed the microscopic structure of zebrafish myocardial tissue by electron microscopy and found that the gap junctions in the myocardial cells of the mutant group were widened; in addition, mitochondrial swelling in the cells was obvious (Supplemental Figure 2c). We also found that a large amount of abnormal substances accumulated in the cells of zebrafish injected with CHMP4C mutants, similar to the structure of apoptotic bodies (Figure 2i). Electron microscopy results showed effects consistent with patients who carried p.S204L and p.E163G mutations, suggesting abnormal autophagy (Figure 2i and Supplemental Figure 2c).

\section{CHMP4C regulates heart development through the autophagy pathway}

To obtain a global view of genes with expression modulated by CHMP4C in zebrafish, we performed 
transcriptome-wide transcription analysis using tissues collected from zebrafish injected with wildtype or p. S204L and p.E163G CHMP4C with RNA-seq. A hierarchical clustering heatmap visualizing this differential expression pattern was provided (Figure 3a), strongly suggesting the effect of CHMP4C p.S204L and p.E163G mutations on gene expression. To determine the biological functions associated with CHMP4C mutations, we performed Gene Ontology (GO) analysis using the DAVID software package. The GO items of the microtubule cytoskeleton and autophagosome membrane were significantly enriched (Figure 3b). CHMP4C is a component of ESCRT-III, a necessary internal fractional complex, and previous studies have shown that its other proteins are associated with autophagy(Metcalf and Isaacs, 2010; Schoneberg, et al., 2018). Therefore, we speculate that the $\mathrm{CHMP}_{4} \mathrm{C}$ mutation affects autophagy and apoptosis.

To assess the effect of $C H M P 4 C$ p.E163G and p.S204L on the above functions, we performed functional validation using HEK293T cells. CHMP $4 C$ wildtype, mutant and control plasmids were transfected into cells using Lipofectamine 2000; after 48 hours, the starved group was treated with serum-free medium (no FBS) for 2 hours, and autophagy-related proteins were then detected. We found that compared with the control and wildtype groups, the mutant groups (p.E163G and p.S204L) exhibited a significant decrease in the autophagy-related protein LC3-I/II (Figure 3c-e). Corresponding changes were also observed for upstream and downstream proteins in the autophagy pathway, such as AKT, p-AKT, mTOR, SQSTM1/p62 and ATG12-ATG5 (Figure 3c). Similar alterations were observed under confocal immunofluorescence microscopy, and decreased autophagy fluorescent particles were observed in the mutant groups (p.E163G and p.S204L), suggesting blockage and impairment of autophagic flow (Figure $3 \mathrm{f}$ and g). Under electron microscopy, a large number of autophagic-like structures were observed in the mutant groups (p.E163G and p.S204L), and autophagic lysosomes were significantly reduced in the mutant groups compared with those in the other two groups (Figure 4a-c), suggesting the presence of an abnormal autophagy pathway. These effects may be caused by the inhibition of autophagy vesicles and lysosome fusion. Additionally, a large number of black, apoptotic body-like high-density substances were observed in the mutant groups, indicating activation of apoptosis.

To further verify the effect of the CHMP4C gene and its mutation on autophagy function, we knocked out the CHMP4C gene in the primary cardiomyocytes of Sprague-Dawley (SD) rats. Primary cardiomyocytes of SD rats were taken 1-3 days after birth. Adeno-chmp4c-crispr/cas9 was added for 72 hours, and the total protein content was extracted from each group. Autophagy-related proteins were then detected. The primary cardiomyocytes showed decreased autophagy, whereas primary cardiomyocyte death increased (Figure $3 \mathrm{~h}$ ), suggesting that CHMP4C plays a very important role in autophagy and that cells lacking CHMP4C will not survive.

We also evaluated the apoptotic signaling pathway in the cells and found that apoptotic activator proteins $(\mathrm{Bax} / \mathrm{Bcl} 2)$ were activated in the mutant groups (p.E163G and p.S204L); corresponding changes were also observed in apoptotic-related proteins, such as caspase 3 and caspase 9 (Figure $4 \mathrm{~d}$ and e). We conclude that CHMP 4 C p.E163G and p.S204L leads to abnormal autophagy, which in turn activates apoptotic signals and impairs myocardial function.

\section{Discussion}

We found a new DCM gene, CHMP $4 C$, and compared mutations in this gene with those in $M Y H 7$, $M Y B P C 3$ and other major genes causing this disease. The clinical symptoms associated with the CHMP4C mutation are relatively mild, and arrhythmia is mostly the first sign. This is consistent with our results in HEK293T cells. That is, autophagy was abnormal under starvation conditions. However, under normal conditions, the cells did not display obvious autophagy dysfunction. Therefore, CHMP4C p.E163G and p.S204L cause insufficiency in cells exposed to stress. Clinically, patients present with a slow process of cardiac insufficiency, and the age of onset is generally later in life (Table 1).

$\mathrm{CHMP} 4 \mathrm{C}$ is a protein of the endosomal sorting complex required for transport, and no related studies have reported its association with heart disease(Arii, et al., 2018; Gatta and Carlton, 2019). ${ }^{20,21}$ CHMP4C plays an important role in regulating the physiological functions of organisms(Thoresen, et al., 2014; Li, et al., 
2015), and previous studies have reported that it may be related to chromosome concentration and cell division(Petsalaki and Zachos, 2018; Carlton, et al., 2012). CHMP4C participates in bud neck polymerization and shears buds, releases luminal vesicles into the inner body cavity, and depolymerizes polymerized ESCRT-III by the Vps4/Vta1 complex for recycling(Petsalaki and Zachos, 2016; Caillat, et al., 2019). We verified the mutation site in a zebrafish model and found that zebrafish expressing these mutant proteins had abnormal cardiac function. We also used HEK293T cells to reveal abnormal autophagy function in CHMP4C-mutant cells; correspondingly, the apoptotic signaling pathway of the cells was activated, providing further confirmation. Previous studies have reported that abnormal aggregation of a subtype of CHMP2B in ESCRT-III leads to autophagic dysfunction, which plays an important role in the regulation of autophagy(Skibinski, et al., 2005). Some researchers have also found that CHMP2B is associated with frontotemporal dementia and amyotrophic lateral sclerosis (ALS), with ubiquitin-positive aggregates present in the brains of patients(Clayton, et al., 2015). One study reported that mutations in CHMP2B can interfere with the fusion of autophagic vacuoles and lysosomes, ultimately leading to autophagosome aggregation(Katzmann, et al., 2003). Recently, it was found that another subunit, CHMP2A, of ESCRT-III regulates the closure of autophagic vacuoles and that abnormal function of this protein leads to abnormal autophagy function in cells(Takahashi, et al., 2018). This indicates that ESCRT-III plays an important role in autophagy. Interestingly, the CHMP4C mutations found in this study were only identified in DCM but not in other types of cardiomyopathy, such as hypertrophic cardiomyopathy (HCM) and arrhythmogenic right ventricular cardiomyopathy (ARVC), suggesting that CHMP4C might be a DCM pathogenic gene.

Autophagy is ubiquitous in eukaryotes. An autophagy induction signal first initiates the formation of a phagocytic bubble. Cleavage of LC3 produces LC3-I, which is then modified to LC3-II, followed by membrane localization and phagocytic extension. The cellular components to be cleared are assembled as mature autophagosomes, which fuse with lysosomes to form autophagosomes, and are then digested, degraded and recovered(Delbridge, et al., 2017). Autophagy is essential for maintaining the stability of cardiac function, and abnormal autophagy in cardiomyocytes can lead to heart disease(Martins-Marques, et al., 2015). Moreover, some researchers have found that cardiomyocyte autophagy leads to cardiac remodeling(Shirakabe, et al., 2016; Heger, et al., 2016). Through the present study, we found that CHMP4C is a new DCM pathogenic gene that can lead to abnormal autophagy function and the clinical phenotype of DCM.

We applied whole-exome sequencing to identify a new gene, CHMP4C, as a pathogenic gene of DCM. The CHMP4C gene is involved in cellular autophagy, and mutation of CHMP4C leads to abnormal autophagy function, resulting in apoptosis activation and cardiac dysfunction in clinical patients. Therefore, CHMP4C should be screened in patients with DCM.

\section{Acknowledgments}

We gratefully acknowledge support from the Shanghai Institute of Medical Imaging and BGI.

We thank Yi Zhang and Juan Shen for providing support with the genetic and bioinformatic analyses. We thank Changjian Gao, Jiaying Hou, Kesu A for their help in electron microscopy and tissue sectioning and Jun Chi for technical support in the zebrafish functional investigation.

\section{Sources of funding}

This work was supported by NSFC Grants (\# 81671685, \#31625013, and \#91732302); Shanghai Science and Technology Committee Foundation (\#17411954400); Shanghai Brain-Intelligence Project from STCSM (16JC1420501); Strategic Priority Research Program of the Chinese Academy of Sciences (XDBS01060200); Program of Shanghai Academic Research Leader, the Open Large Infrastructure Research of Chinese Academy of Sciences; and the Shanghai Municipal Science and Technology Major Project (\#2018SHZDZX05).

\section{Disclosure}

None. 


\section{References:}

Arii J, Watanabe M, Maeda F, Tokai-Nishizumi N, Chihara T, Miura M,... Kawaguchi Y. 2018. ESCRT-III mediates budding across the inner nuclear membrane and regulates its integrity. Nat Commun 9(1):3379.

Bagnall RD, Ingles J, Semsarian C. 2011. Molecular diagnostics of cardiomyopathies: the future is here. Circ Cardiovasc Genet 4(2):103-4.

Bollen I, Schuldt M, Harakalova M, Vink A, Asselbergs FW, Pinto JR,... van der Velden J. 2017. Genotypespecific pathogenic effects in human dilated cardiomyopathy. J Physiol 595(14): 4677-4693.

Caillat C, Maity S, Miguet N, Roos WH, Weissenhorn W. 2019. The role of VPS4 in ESCRT-III polymer remodeling. Biochem Soc Trans 47(1):441-448.

Carlton JG, Caballe A, Agromayor M, Kloc M, Martin-Serrano J. 2012. ESCRT-III governs the Aurora B-mediated abscission checkpoint through CHMP4C. Science 336(6078):220-5.

Charron P, Arad M, Arbustini E, Basso C, Bilinska Z, Elliott P,... Tavazzi L. 2010. Genetic counselling and testing in cardiomyopathies: a position statement of the European Society of Cardiology Working Group on Myocardial and Pericardial Diseases. Eur Heart J 31(22):2715-26.

Clayton EL, Mizielinska S, Edgar JR, Nielsen TT, Marshall S, Norona FE,... Isaacs AM. 2015. Frontotemporal dementia caused by CHMP2B mutation is characterised by neuronal lysosomal storage pathology. Acta Neuropathol 130(4):511-23.

Costa MC. 2019. Genomic characterization in dilated cardiomyopathy. Rev Port Cardiol 38(6):449-450.

Delbridge L, Mellor KM, Taylor DJ, Gottlieb RA. 2017. Myocardial stress and autophagy: mechanisms and potential therapies. Nat Rev Cardiol 14(7):412-425.

Dellefave L, McNally EM. 2010. The genetics of dilated cardiomyopathy. Curr Opin Cardiol 25(3):198-204.

Gatta AT, Carlton JG. 2019. The ESCRT-machinery: closing holes and expanding roles. Curr Opin Cell Biol 59:121-132.

Gentile P, Merlo M, Cannata A, Gobbo M, Artico J, Stolfo D,... Sinagra G. 2019. Dilated Cardiomyopathy With Mid-Range Ejection Fraction at Diagnosis: Characterization and Natural History. J Am Heart Assoc 8(17):e010705.

Han H, Hill CP. 2019. Structure and mechanism of the ESCRT pathway AAA+ ATPase Vps4. Biochem Soc Trans 47(1):37-45.

Heger J, Schulz R, Euler G. 2016. Molecular switches under TGFbeta signalling during progression from cardiac hypertrophy to heart failure. Br J Pharmacol 173(1):3-14.

Hershberger RE, Lindenfeld J, Mestroni L, Seidman CE, Taylor MR, Towbin JA. 2009. Genetic evaluation of cardiomyopathy-a Heart Failure Society of America practice guideline. J Card Fail 15(2):83-97.

Hershberger RE, Siegfried JD. 2011. Update 2011: clinical and genetic issues in familial dilated cardiomyopathy. J Am Coll Cardiol 57(16):1641-9.

Katzmann DJ, Stefan CJ, Babst M, Emr SD. 2003. Vps27 recruits ESCRT machinery to endosomes during MVB sorting. J Cell Biol 162(3):413-23.

Kimura A. 2016. Molecular genetics and pathogenesis of cardiomyopathy. J Hum Genet 61(1):41-50.

Lakdawala NK, Winterfield JR, Funke BH. 2013. Dilated cardiomyopathy. Circ Arrhythm Electrophysiol 6(1):228-37.

Lee JA, Beigneux A, Ahmad ST, Young SG, Gao FB. 2007. ESCRT-III dysfunction causes autophagosome accumulation and neurodegeneration. Curr Biol 17(18):1561-7. 
Li K, Liu J, Tian M, Gao G, Qi X, Pan Y,... Su X. 2015. CHMP4C Disruption Sensitizes the Human Lung Cancer Cells to Irradiation. Int J Mol Sci 17(1).

Martins-Marques T, Ribeiro-Rodrigues T, Pereira P, Codogno P, Girao H. 2015. Autophagy and ubiquitination in cardiovascular diseases. DNA Cell Biol 34(4):243-51.

McNally EM, Golbus JR, Puckelwartz MJ. 2013. Genetic mutations and mechanisms in dilated cardiomyopathy. J Clin Invest 123(1):19-26.

Metcalf D, Isaacs AM. 2010. The role of ESCRT proteins in fusion events involving lysosomes, endosomes and autophagosomes. Biochem Soc Trans 38(6):1469-73.

Pasotti M, Klersy C, Pilotto A, Marziliano N, Rapezzi C, Serio A,... Arbustini E. 2008. Long-term outcome and risk stratification in dilated cardiolaminopathies. J Am Coll Cardiol 52(15):1250-60.

Petsalaki E, Dandoulaki M, Zachos G. 2018. Chmp4c is required for stable kinetochore-microtubule attachments. Chromosoma 127(4):461-473.

Petsalaki E, Zachos G. 2016. Clks 1, 2 and 4 prevent chromatin breakage by regulating the Aurora Bdependent abscission checkpoint. Nat Commun 7:11451.

Petsalaki E, Zachos G. 2018. CHMP4C: A novel regulator of the mitotic spindle checkpoint. Mol Cell Oncol 5(3):e1445944.

Pike T, Brownlow N, Kjaer S, Carlton J, Parker PJ. 2016. PKCvarepsilon switches Aurora B specificity to exit the abscission checkpoint. Nat Commun 7:13853.

Reichart D, Magnussen C, Zeller T, Blankenberg S. 2019. Dilated cardiomyopathy: from epidemiologic to genetic phenotypes: A translational review of current literature. J Intern Med 286(4):362-372.

Rusten TE, Stenmark H. 2009. How do ESCRT proteins control autophagy? J Cell Sci 122(Pt 13):2179-83.

Schoneberg J, Pavlin MR, Yan S, Righini M, Lee IH, Carlson LA,... Hurley JH. 2018. ATP-dependent force generation and membrane scission by ESCRT-III and Vps4. Science 362(6421):1423-1428.

Shirakabe A, Ikeda Y, Sciarretta S, Zablocki DK, Sadoshima J. 2016. Aging and Autophagy in the Heart. Circ Res 118(10):1563-76.

Skibinski G, Parkinson NJ, Brown JM, Chakrabarti L, Lloyd SL, Hummerich H,... Collinge J. 2005. Mutations in the endosomal ESCRTIII-complex subunit CHMP2B in frontotemporal dementia. Nat Genet 37(8):806-8.

Takahashi Y, He H, Tang Z, Hattori T, Liu Y, Young MM,... Wang HG. 2018. An autophagy assay reveals the ESCRT-III component CHMP2A as a regulator of phagophore closure. Nat Commun 9(1):2855.

Thoresen SB, Campsteijn C, Vietri M, Schink KO, Liestol K, Andersen JS,... Stenmark H. 2014. ANCHR mediates Aurora-B-dependent abscission checkpoint control through retention of VPS4. Nat Cell Biol 16(6):550-60.

Watkins H, Ashrafian H, Redwood C. 2011. Inherited cardiomyopathies. N Engl J Med 364(17):1643-56.

Worman HJ, Bonne G. 2007. "Laminopathies": a wide spectrum of human diseases. Exp Cell Res 313(10):2121-33.

\section{Hosted file}

figure.docx available at https://authorea.com/users/301649/articles/431494-mutations-inchmp4c-cause-dilated-cardiomyopathy-via-dysregulation-of-autophagy 